\title{
ANXIETY DISORDERS - CLINICAL AND NEUROBIOLOGICAL ASPECTS
}

\section{ANGSTSTÖRUNGEN - KLINISCHE UND NEUROBIOLOGISCHE ASPEKTE}

\author{
Theresa Lahousen \& Hans-Peter Kapfhammer \\ Klinik für Psychiatrie und Psychotherapeutische Medizin, Medizinische Universität Graz, Graz, Austria
}

\begin{abstract}
SUMMARY
Anxiety disorders are considered among the most common psychiatric disorders in general population. They may be characterized by prominent subjective suffering, frequent chronic courses of illness, increased rates of comorbid other psychiatric disorders and somatic diseases, a distressing amount of psychosocial disabilities, in all, a challenging high burden of disease.

Anxiety disorders have principally to be conceptualized within a multifactorial biopsychosocial model. Various psychological and psychosocial approaches have contributed to a multi-layered understanding of various major predisposing, eliciting, and maintaining factors in the course of illness. Modern neurobiological research has significantly broadened and deepened the aetiopathogenetic complexity of anxiety disorders. The main focus of this short review is on neural fear-and anxiety circuits, neurotransmitter systems, neuroendocrine and inflammatory stress systems, genetics and epigenetics that characterize the general basis of fear and anxiety regulation and their dysregulation in anxiety disorders.

Anxiety disorders may be effectively treated both by psychotherapeutic and pharmacological approaches. Basic principles and general guidelines in the treatment of anxiety disorders are being presented.
\end{abstract}

Key words: anxiety disorders - epidemiology - course of illness - psychology - neurobiology - fear-anxiety circuits neurotransmitter - stress systems - genetics - epigenetics

\section{EINLEITUNG}

Angst zählt zu den Basisemotionen des Menschen. Das Angstsystem ist hierbei tief in der Phylogenese verankert und als evolutionäres Erbe dem Säugling bereits von Geburt an verfügbar (Montag \& Panksepp 2017). Angst spielt als primäres biosoziales Signal in der frühen Mutter-Kind-Dyade eine zentrale Rolle. Sie trägt entscheidend zur Qualität von zwischenmenschlicher Bindung und risikobewusstem Umgang mit der Außenwelt bei. Angst drückt stets einen Zustand von Bedrohung aus. Fortlaufende Lernprozesse prägen sowohl die differentielle Wahrnehmung als auch die kognitive Bewertung von inneren und äußeren Gefahren. Sie

\section{ZUSAMMENFASSUNG}

Angststörungen zählen zu den häufigsten psychischen Störungen in der Allgemeinbevölkerung. Sie bedeuten hohes subjektives Leiden und verlaufen oft chronisch. Sie sind mit zusätzlichen komorbiden psychischen und auch mit somatischen Krankheitsrisiken verbunden. Sie verursachen zahlreiche psychosozialen Beeinträchtigungen und große sozioökonomische Belastungen.

Angststörungen werden heute prinzipiell innerhalb eines multifaktoriellen, biopsychosozialen Krankheitsmodells konzipiert. Psychologische und psychosoziale Modelle haben zu einem sehr differenzierten ätiopathogenetischen Verständnis der Einflüsse auf Entstehung, Auslösung und Aufrechterhaltung von Angststörungen geführt, das durch moderne neurobiologische Forschungsergebnisse noch signifikant erweitert und vertieft worden ist.

Angststörungen können sowohl psychopharmakologisch als auch psychotherapeutisch wirksam behandelt werden. In einer Gesamtbeurteilung kann die große Mehrheit der unkomplizierten spezifischen Phobien und Panikstörungen gut und sehr wahrscheinlich auch anhaltend mit Psychotherapie gebessert werden. Generalisierte Angststörungen und soziale Phobien benötigen hingegen häufig längerfristige störungsorientierte Psychotherapien. Schwere Angststörungen aber, vor allem bei chronischen Verläufen, mit zusätzlichen psychischen und somatischen Komplikationen und ausgeprägter psychosozialer Beeinträchtigung werden in der Regel bevorzugt medikamentös und oft auch in Kombination mit Psychotherapie behandelt.

Schlüsselwörter: Angststörungen - Epidemiologie - Verlauf - psychologische Modelle - neurobiologische Modelle Furcht-Angst-Regelkreis - Neurotransmitter - Stresssysteme Genetik - Epigenetik bestimmen schließlich das erreichte Ausmaß des individuell tolerierten Angstniveaus und beeinflussen dominante Verhaltensstile, um wieder Sicherheit und Schutz für sich herzustellen. Angst manifestiert sich auf einer subjektiven Ebene in Form bestimmter Kognitionen (z. B. Gefahr, Katastrophe, Beschämung) und Befürchtungen, auf einer physiologischen Ebene in Form körperlicher Reaktionen (z. B. autonom-nervöse, meist dominant sympathikotone Aktivierung, Stresshormonantwort), auf einer motorischen Ebene in Form definierter Verhaltensweisen (z. B. Fliehen, Vermeiden, Erstarren, Kämpfen). Angst ist in dieser Perspektive als ein normales psychobiologisches Phänomen von hoher adaptiver Relevanz anzusehen. 
Potentielle Krankheitswertigkeit erlangt Angst entweder durch ein Zuviel oder ein Zuwenig in der Intensität und Häufigkeit ihres Auftretens. Als psychopathologisches Symptom kommt Angst bei fast allen psychischen Störungen vor (Oyebode 2014). Angststörungen verweisen als eigenständige diagnostische Gruppe auf Ängste und Befürchtungen, deren Ausmaß, Gefahrenattribution und/oder Persistenz unverhältnismäßig stark ausgeprägt sind, im subjektiven Urteil als unrealistisch eingestuft werden können, hierbei mit einem starken Leidensdruck und bedeutsamen psychosozialen Beeinträchtigungen einhergehen. Angststörungen treten komorbid oft mit anderen psychischen Störungen wie z.B. mit depressiven oder Substanz-bezogenen Störungen auf. Sie gehen auch mit zahlreichen somatischen Erkrankungen wie z.B. mit Herz- und Kreislauferkrankungen einher (Kapfhammer 2017).

\section{KLASSIFIKATORISCHE EINORDNUNG DER ANGSTSTÖRUNGEN}

Das aktuelle psychiatrische Klassifikationssystem von DSM-5 und die künftige ICD-11 verhalten sich in der Beschreibung der diagnostischen Gruppe der Angststörungen konzeptuell sehr ähnlich zueinander (Kogan et al. 2016, Craske et al. 2017). Im Vergleich zu ihren jeweiligen Vorgängerversionen sind zwei prinzipielle Erneuerungen festzuhalten: Sowohl die akuten Belastungs- und posttraumatischen Störungen als auch die Zwangsstörungen sind jeweils in eigenständige, aber benachbarte diagnostische Gruppen ausgegliedert worden. In beiden Klassifikationssystemen weist die diagnostische Gruppe der Angststörungen eine grundlegende Unterscheidung nach einem objekt- und/oder situationsabhängigen versus objekt- und/oder situationsunabhängigem Auftreten auf und betont bei letzterer Manifestation eine Differenzierung nach akuter anfallsartiger versus chronischer generalisierter Klinik. Als einzelne Angststörungen werden spezifische (isolierte) phobische Störungen, als spezielle Varianten die soziale Phobie und die Agoraphobie, sowie die Panikstörung und die generalisierte Angststörung beschrieben. In beiden Klassifikationssystemen wird für eine definitive Diagnosestellung nicht nur das Vorliegen typischer psychopathologischer Merkmale der jeweiligen Angststörung verlangt, sondern auch ein Schweregrad der Symptome gefordert, der in persönlichen, interpersonalen, sozialen und beruflichen Funktionsbereichen zu erheblichen Belastungen oder Beeinträchtigungen geführt hat.

\section{KLINISCHE TYPISIERUNG DER EINZELNEN ANGSTSTÖRUNGEN}

Den spezifischen Phobien ist eine irrationale Furcht vor bestimmten Situationen oder Objekten gemeinsam, sodass ein heftiges Bedürfnis nach einem sicherheitsstiftenden Vermeidungsverhalten entsteht. Über die Vermeidung kann eine Kontrolle der umschriebenen situa- tiven oder Objekt-gerichteten Furcht wieder erlangt werden. $\mathrm{Zu}$ den spezifischen (isolierten) Phobien wird üblicherweise nicht die Furcht vor Angstanfällen, d.h. vor einem Wiederauftreten von Panikattacken und auch nicht vor definierten sozialen Situationen gezählt. In der unmittelbaren Konfrontation mit einem definierten phobischen Objekt oder einer spezifisch gefürchteten Situation kann das Angstniveau aber durchaus ein panikartiges Ausmaß annehmen. Die häufigsten spezifischen (isolierten) Phobien beziehen sich auf Tiere, enge Räume, Höhen, Flugzeuge, auf gefährliche Gegenstände, Blut, körperliche Verletzungen, auf medizinische Utensilien und Orte. Bei krankheitsbezogenen Phobien, wie z. B. der Karzinophobie führt die Vermeidungshaltung oft nicht mehr zu einer wirksamen Angstkontrolle. Übergänge dieser Krankheitsphobien zur Hypochondrie sind fließend. Unterschiedliche Schweregrade der spezifischen (isolierten) Phobien mit im Extremfall ausgeprägtem Leiden und starker Alltagsbeeinträchtigung sind möglich (Eaton et al. 2018).

Die soziale Phobie wird diagnostisch eigenständig bewertet. Sie wird durch eine unangemessene, häufig dauerhafte Furcht vor und Vermeidung von sozialen Situationen charakterisiert, in denen Menschen sich einer interpersonalen Bewertung ausgesetzt fühlen. Angst vor leistungsbezogenem Versagen, vor sozialer Beschämung und Demütigung sind typisch. Soziale Ängste können eng umschrieben (z. B. vor öffentlichem Sprechen) sein oder aber als soziale Angststörung generalisiert fast alle zwischenmenschlichen Kontakte und Aktivitäten beherrschen. Eine ausgeprägte Erwartungsangst bei bevorstehender Konfrontation mit einer bestimmten sozialen Situation ist kennzeichnend. In der jeweiligen sozialen Situation kann das Angstniveau wiederum bis zur unbeherrschbaren Panik ansteigen (Leichsenring \& Leweke 2017).

Die Agoraphobie beschreibt ein dominantes Vermeidungsverhalten gegenüber Situationen, in denen das Auftreten von Angstattacken befürchtet wird. Ursprünglich bedeutet Agoraphobie eine Angst vor öffentlichen Plätzen. Sie bezieht sich meist auf Orte mit größeren Menschenansammlungen. Typisch ist hierbei aber weniger die Angst vor diesen Orten als solchen, sondern vielmehr die Angst, fern einer vertrauten häuslichen Umgebung in eine hilflose Situation geraten zu können. Eine Generalisierung des Vermeidungsverhaltens wird im weiteren Verlauf häufig beobachtet. Sie führt dann $\mathrm{zu}$ einer grundlegenden Veränderung in den bisherigen Lebensgewohnheiten und bedingt einen sozialen Rückzug mit ausgeprägten Behinderungsgraden. Agoraphobie ist sehr häufig eine Folge von Panikattacken. Sie kann sich aber auch ohne solche vorangehenden Angstattacken entwickeln (Asmundson et al. 2014).

Die Panikstörung zeichnet sich durch plötzliche, d. h. ohne unmittelbar erkennbare äußere. Gefahren auftretende Angstanfälle in bestimmter Häufigkeit und zeitlicher Dauer und ohne eine diagnostizierbare somatische Ursache aus. Eine ausgeprägte Besorgnis vor wiederkehrenden Angstanfällen resultiert. Typisches formales 
Merkmal ist das crescendohafte Eskalieren der Angstintensität in einer Panikattacke. Das Paniksyndrom wird durch eine Vielzahl somatischer Symptome charakterisiert. Sie kann von Todesangst, von einem Gefühl des Kontrollverlusts, einer unmittelbar bevorstehenden seelischen und körperlichen Katastrophe, von irritierender Depersonalisation und Derealisation bestimmt sein. Panikattacken und die sich entwickelnde Angst vor der Angst (,Phobophobie“) ziehen fast regelhaft ein starkes Vermeidungsverhalten nach sich (Lock et al. 2015).

Bei der Generalisierten Angststörung (GAD) imponiert wiederum ein anhaltend erhöhtes Angstniveau, meist ohne beherrschende Paniksymptome, jedoch mit zentralen unrealistischen Besorgnissen oder übertriebenen Katastrophenerwartungen. Symptome einer muskulären Verspannung, einer autonom-nervösen Hyperaktivität und Hypervigilanz im Umweltbezug definieren das klinische Bild. Die diagnostische Konstruktion der GAD verweist per se auf eine inhärente Chronizität ihres Verlaufs (Goodwin et al. 2017).

Als weitere Angst-bedingte Störungen werden in der Kategorie der Angststörungen für das Erwachsenenalter gemäß DSM-5 Angststörungen infolge einer medizinischen Krankheitsbedingung sowie als induziert durch Substanzen bzw. Medikamenten, für das Kindesalter die Trennungsangststörung und der selektive Mutismus aufgeführt. In der ICD-11 wird weiterhin auch eine gemischte depressive und Angststörung kodierbar sein.

\section{EPIDEMIOLOGISCHE ASPEKTE DER ANGSTSTÖRUNGEN}

Angststörungen zählen zu den häufigsten psychischen Störungen in der Allgemeinbevölkerung (Stein et al. 2017). Dies bestätigt auch die derzeit aktuellste Studie zu den 1-Jahres-Prävalenzen von psychischen Erkrankungen in der Europäischen Union sowie Schweiz, Island und Norwegen. Angsterkrankungen stehen unverändert mit einer 1-Jahres- Prävalenz von $14 \%$ an der Spitze der Häufigkeitspyramide und betreffen derzeit ca. 61,5 Mio. Personen in der definierten Untersuchungsregion (Wittchen et al. 2011). Eine Metaanalyse aus weltweit 63 Ländern zur Prävalenz psychischer Erkrankungen zeigt für Angststörungen eine Lebenszeitprävalenz von $12,9 \%$ an. Frauen weisen mit 18,2 \% hierbei ein ca. doppelt so hohes Erkrankungsrisiko wie die Männer mit 10,1 \% auf (Steel et al. 2014).

In den meisten epidemiologischen Untersuchungen rangieren die spezifischen Phobien an der Häufigkeitsspitze. Die Prävalenzraten in der Allgemeinbevölkerung werden für die Lebenszeitperspektive zwischen 8,8\% und 12,5\% angegeben (Kessler et al. 2005; Stinson et al. 2007). Für die Panikstörung wird eine durchschnittliche Lebenszeitprävalenz zwischen $2 \%$ und $5 \%$ berichtet. Hinsichtlich Persistenz, Anzahl der Attacken in der Lebenszeit, Anzahl der Jahre mit Attacken, klinischem Schweregrad muss ein Kontinuum angenommen werden (Kessler et al. 2005). Die Agoraphobie steht in einem besonderen konditionalen Verhältnis zur Panikstörung. Sie kann sich aber auch isoliert ohne vorhergehende Angstanfälle entwickeln. Während eine frühere europäische Übersicht eine relativ hohe Häufigkeit von 3,5\% feststellte (Wittchen et al. 1998), deckte eine nachfolgende US-amerikanische Studie nur eine relativ niedrige Rate von $0,2 \%$ Lebenszeitprävalenz auf (Grant et al. 2006). Auch die soziale Phobie weist hohe Prävalenzraten auf. Sie zählt möglicherweise neben den spezifischen Phobien zu den häufigsten Einzelangststörungen. Hierbei haben aber sowohl veränderte diagnostische Kriterien in den sukzessiven Diagnosemanualen als auch jeweils ausgewählte unterschiedliche Erfassungsmethoden große Auswirkungen auf die in einzelnen epidemiologischen Untersuchungen ermittelten Häufigkeitsraten. So ergab beispielsweise die mittels CIDI durchgeführte NCS Replikationsstudie eine Lebenszeitprävalenz von $12,8 \%$ (Kessler et al. 2005), hingegen der auf strukturierten klinischen Interviews basierende National Epidemiologic Survey on Alcohol and Related Conditions mit 5\% eine deutlich niedrigere Lebenszeitprävalenz (Grant et al. 2005). Die 1- JahresPrävalenz für die generalisierte Angststörung betrug in der NCS-Replikationsstudie 3,1\% und die Lebenszeitprävalenz 5,7 \% (Kessler et al. 2005). Einer Metaanalyse zufolge streuten die Lebenszeitprävalenzen in europäischen Studien im Vergleich hierzu zwischen 0,1\% und $6,9 \%$ (Lieb et al. 2005). Die vermutlich aussagekräftigste ESEMeD-Untersuchung unter den in die Metaanalyse eingeschlossenen Studien ermittelte eine Rate von 2,8 \% (ESEMeD/MHEDEA 2000 Investigators 2004). Generell kann auch für die einzelnen Angststörungen ein je ungefähr 2-fach erhöhtes Erkrankungsrisiko zuungunsten der Frauen angenommen werden (Wittchen et al. 2011).

Innerhalb des medizinischen Versorgungssystems weisen Patienten mit definierten Angststörungen möglicherweise ein differentielles Inanspruchnahmeverhalten auf. So suchen Patienten mit Panikstörung wegen der prominenten und als besonders beunruhigend erlebten körperlichen Paniksymptome besonders als Notfallpatienten diverse Fachärzte und somatische Spezialambulanzen auf, wie z.B. Neurologen (Kopfschmerz, Schwindel), Kardiologen (Brustschmerz, Tachykardie) oder Gastroenterologen (Übelkeit, Colon irritabile). Eine Panikstörung liegt sehr häufig sog. ,,medizinisch unerklärten Körpersymptomen" zugrunde und kann ein exzessives Inanspruchnahmeverhalten medizinischer Einrichtungen bedingen. Die hiermit verbundenen sozioökonomischen Kosten sind beträchtlich (Tolin et al. 2009). Patienten mit einer generalisierten Angststörung werden wiederum v. a. von Familien- bzw. Hausärzten betreut, wie eine WHO Collaborative Study beschrieb (Sartorius et al. 1996). Im Vergleich hierzu werden Patienten mit einer sozialen Phobie wesentlich seltener auf den unterschiedlichen Ebenen des medizinischen Versorgungssystems in ihrer speziellen Angstproblematik erkannt. Die Zeitspanne bis zu einer korrekten Diagnosestellung beträgt weit über 10 Jahre und führt in 
einem signifikant geringeren Prozentsatz dann auch zu angemessenen psychopharmakologischen und/oder psychotherapeutischen Interventionen (Rees et al. 1998). Sehr selten werden auch Patienten mit spezifischen Phobien im ärztlichen Versorgungssystem vorstellig. Eine wichtige Ausnahme stellen aber jene Fälle mit krankheits-spezifischen Phobien, oder mit einem angemessenen Krankheitsverhalten negativ interferierende Blut-, Verletzungs- oder speziell auf den Schluckvorgang bezogene Phobien dar (McNally 1994, Cisler et al. 2009).

Einzelne Angsterkrankungen zeigen hinsichtlich des Ersterkrankungsalters ein differenzielles Muster: Panikstörung, Agoraphobie: zwischen dem 20. und 30. Lebensjahr; generalisierte Angststörung: bimodal sowohl späte Adoleszenz und junges Erwachsenenalter als auch ab 40. Lebensjahr; soziale Phobie: Adoleszenz; spezifische Phobien: Kindheit und frühe Adoleszenz (Kessler et al. 2009). In 80-90\% der Fälle beginnen Angststörungen vor dem 35. Lebensjahr. Die Zeit zwischen dem 10. und 25. Lebensjahr ist als ein Hochrisikoabschnitt für die Entwicklung einer Angststörung anzusehen. Jenseits des 4. Lebensjahrzehnts sinkt das Risiko einer Ersterkrankung bedeutsam - mit Ausnahme der generalisierten Angststörung, die als einzige Angststörung im Alter eine erhöhte Prävalenz aufweist (Lijster et al. 2017, Canuto et al. 2018). Die im DSM-5 neu in der Kategorie der Angsterkrankungen aufgeführte „Trennungsangststörung“" ist durch eine anhaltende, exzessive und entwicklungspsychologisch inadäquate Trennungsangst von wichtigen Bezugspersonen gekennzeichnet. Die Lebenszeitprävalenz dieser meist im Kindesalter erstdiagnostizierten Angststörung liegt bei ca. $5 \%$ (Shear et al. 2006). Die Bedeutung der Trennungsangst für die Entwicklung von anderen Angsterkrankungen, insbesondere der Panikstörung im Erwachsenenalter wurde metaanalytisch eindrucksvoll nachgewiesen (Kossowsky et al. 2013).

Angststörungen nehmen in der sozioökonomischen Perspektive des „Burden of Disease“ einen höchst bedeutsamen Rang hinsichtlich der sog. Years Lived with Disability (YLD), also der mit Behinderung bzw. der mit chronischer Erkrankung gelebten Jahre ein. Hinsichtlich Disability-Adjusted Life Years (DALY), einem kombinierten Maß für die Anzahl der durch frühe Sterblichkeit oder Beeinträchtigung bei einer Erkrankung verlorenen Lebensjahre, aber auch hinsichtlich direkter und indirekter Kosten liegen Angsterkrankungen in der Spitzengruppe der sozioökonomisch relevantesten Erkrankungen (Murray et al. 2013, Patel et al. 2016, Craske et al. 2017).

\section{VERLAUF DER ANGSTERKRANKUNGEN}

Nicht nur die Daten aus epidemiologischen Studien, welche die Angsterkrankungen als die psychischen Störungen mit der weitesten Verbreitung in der Allgemeinbevölkerung kennzeichnen, sondern v. a. die Erkenntnis über ihre häufige Chronizität mit einer damit assoziierten bedeutsamen psychischen Komorbidität bzw. als Vorläufererkrankungen insbesondere für affektive und Substanz-bedingte Störungen und hieraus resultierenden beträchtlichen psychosozialen Behinderungsgraden unterstreichen den klinischen und gesundheitspolitischen Stellenwert der Angststörungen. Eine zusätzliche Bestärkung dieser Einschätzung zeigt sich in der ebenso bedeutsamen, aber im medizinischen Versorgungssystem zu selten eigenständig reflektierten hohen Komorbiditätsrate mit zahlreichen somatisch-medizinischen Erkrankungen im weiteren Verlauf. Hierbei sind bidirektionale Wechselwirkungen in komplexen psychosomatischen und somato-psychischen Modellen zu diskutieren und jeweils reziproke negative Auswirkungen und Komplizierungen des gesamten Krankheitsverlaufs zu beachten (Kapfhammer 2017, 2018). Wichtige differentielle Verlaufsaspekte müssen bei den einzelnen speziellen Angststörungen beachtet werden (Domschke et al. 2017).

\section{ÄTIOPATHOGENETISCHE MODELL MIT FOKUS AUF ALLGEMEINE NEUROBIOLOGISCHE ASPEKTE BEI ANGSTSTÖRUNGEN}

Verursachung, Auslösung und Verlauf von Angststörungen werden heute innerhalb eines multifaktoriellen biopsychosozialen Krankheitsmodells konzipiert. Weiterhin zentrale Bedeutung können die psychologischen und psychosozialen Modellvorstellungen für das Verständnis von Angststörungen beanspruchen. Empirische Forschungsergebnisse aus der Lernpsychologie, der psychodynamischen und Traumapsychologie, der Entwicklungspsychologie und Bindungstheorie, der Temperaments- und Persönlichkeitspsychologie haben mittlerweile zu einem höchst differenzierten Bild über die mannigfaltigen psychosozialen und psychologischen Bedingungsfaktoren zu früh erworbenen Dispositionen und Vulnerabilitäten, über wichtige Auslösefaktoren der einzelnen Angststörungen und über entscheidende Einflussfaktoren auf deren Aufrechterhaltung in differentiellen Verläufen geführt (Domschke et al. 2017). Psychologische und psychosoziale Forschungsansätze haben zudem die Basis für zahlreiche störungsorientierte Psychotherapieansätze gelegt, die sich durch empirische Nachweise einer hohen Wirksamkeit auszeichnen. Diese nehmen in der Behandlung von Angstpatienten innerhalb des fachärztlichen, klinisch-psychologischen und psychotherapeutischen Versorgungssystems eine zentrale Rolle ein (Craske et al. 2017).

Während der zurückliegenden Jahrzehnte wurden die ätiopathogenetischen Zusammenhänge bei Angststörungen durch zahlreiche Erkenntnisse mittels unterschiedlicher Methoden der neurobiologischen Forschung signifikant erweitert. Es sollen nur einige allgemeine neurobiologische Aspekte bei Angststörungen hier skizziert werden (Domschke et al. 2017). 
Allgemeine neurobiologische Modelle der Angststörungen konzentrieren sich auf neuronale Regelkreise zwischen distinkten neuroanatomischen Strukturen, denen eine grundlegende Funktion in der Entstehung und Vermittlung von Angstreaktionen zukommt. Sie favorisieren ferner eine Dysfunktionalität in definierten Neurotransmittersystemen und zugehörigen Rezeptorpopulationen.

Erkenntnisse aus stressinduzierten Veränderungen in diesen Systemen und assoziierten neuroendokrinen und neuroinflammatorischen Mechanismen, aber auch die mittlerweile vorliegenden genetischen und epigenetischen Befunde haben zu einem tieferen Verständnis der Neurobiologie von Angst und Angststörungen beigetragen.

\section{Neuroanatomische Modelle der Angststörungen}

Mehrere neuroanatomische Regionen sind in der Regulation von Aufmerksamkeit, Arousal, Stresserleben und Angst involviert. Arousal ist nicht mit Angst gleichzusetzen, trägt aber zu ihrer Entstehung bedeutsam bei. Das Niveau des Arousals wird von Zentren im Hirnstamm kontrolliert, die auch wichtig für die Entstehung von Angst sind. Hierzu zählen der noradrenerge Locus coeruleus, die serotonergen Raphekerne und der $\mathrm{Nu}$ cleus paragigantocellularis mit noradrenergen, serotonergen, cholinergen und glutamatergen Rezeptoren. Die besondere Rolle des Locus coeruleus in der Vigilanz und Signalverarbeitung ist hervorzuheben. Er ist nicht die primäre Quelle der Angstentstehung, dient aber als ein entscheidendes Amplifikationssystem (Geiger et al. 2014). Die primären Emotionen von Angst und Furcht werden entscheidend im limbischen System (Amygdala, Bettkern der Stria terminalis, Hippocampus, Nuclei septi, Hypothalamus) organisiert. Neuere Forschungen favorisieren eine Differenzierung in „Furchterkrankungen“" (,phasic fear") einerseits, charakterisiert durch eine schnell an- und wieder abflutende Furcht vor klar spezifizierten Stimuli, und „Angsterkrankungen“ (,sustained fear") andererseits, gekennzeichnet durch eher langandauernde Angst bzw. antizipatorische Angstanspannung und ausgelöst durch eher unspezifische und nur schwer vorhersagbare Stimuli (Davis et al. 2010). In dieser Forschungsperspektive käme der Amygdala eine zentrale Rolle als Schaltstelle der Furchtentstehung zu, dem angrenzenden Bettkern der Stria terminalis hingegen eine bevorzugte Bedeutung in der Generierung und Vermittlung von Angst zu (LeDoux \& Pine 2016). Beide neuronale Strukturen sind aber nicht nur neuroanatomisch eng benachbart, sondern auch funktionell intensiv miteinander verschaltet. Grundsätzlich werden zwei evolutionär erworbene Notfallschaltkreise bei drohenden Gefahren nachgewiesen. Diese unterscheiden sich in der Geschwindigkeit, aber auch in der kognitiven Differenziertheit der Gefahren-bezogenen Informationsverarbeitung:

- Ein rascher Pfad leitet über den Thalamus sensorische Informationen über Gefahren unter Umgehung kortikaler Strukturen direkt zur Amygdala, wo ein Furchtaffekt generiert wird. Die baslolateralen Anteile der Amygdala sind grundlegend für die
Detektion von Gefahrenreizen, die emotionale Konditionierung und Gedächtnisbildung sowie für die autonome Aktivierung und Expression von Affektspannung. Der zentrale Nukleus der Amygdala ist wiederum mit einer Reihe subkortikaler Zentren verschaltet, die einfache, aber differenzierte autonome, endokrine und motorische Abwehrreaktionen auslösen (Ulrich-Lai \& Herman 2009): Locus coeruleus: Blutdruck- und Pulsanstieg; Nucleus brachialis: Erhöhung der Atemfrequenz; Nucleus paraventricularis des Hypothalamus: über $\mathrm{CRH}$ die Aktivierung der HPA-Stresshormon-Achse; Nucleus lateralis des Hypothalamus: Aktivierung des sympathischen Nervensystems; motorischer Kortex: Planung und Ausführung von motorischen Reaktionen ohne vollständige Analyse der Gefahrensituation; periaquäduktales Grau: Induktion von Freezingverhalten und Totstellreflex; dorsaler Vaguskern: Aktivierung des parasympathischen Nervensystems. In einem engen funktionellen Zusammenspiel von Amygdala und Hippocampus kann eine Gefahr mit früheren Erfahrungen verglichen bzw. kontextualisiert werden. Von großer klinischer Bedeutung ist, dass eine amygdaläre Hyperaktivität einerseits mit einer Reduktion des ökonomisierenden und modulierenden Effektes des präfrontalen Kortex einhergeht, andererseits auch die kognitiv-mnestische Leistungsfähigkeit des Hippocampus beeinträchtigen kann (Arnsten 2009).

- Ein langsamerer Pfad leitet eintreffende Informationen über den Thalamus zu den primären und anschließend zu den sekundären sensorischen Kortexarealen weiter. Ein Vergleich mit Vorerfahrungen, die nach Sinnesmodalitäten unterschiedlich gespeichert sind, findet statt. Diese kognitive Prozessierung wird wieder an den Hippocampus zurückgeführt und dient hier einer kognitiv höher strukturierten Analyse der aktuellen Gefahr. Die Aktivität der Amygdala unterliegt unter normalen Umständen bedeutsamen modulierenden Einflüssen des präfrontalen Kortex (PFC), der Informationen aus zahlreichen Hirnarealen integriert. Seine grundlegenden Funktionen bestehen in der Bewertung, Planung, Entscheidung und Modulation von Verhaltensweisen, nicht aber in der Vermittlung von automatischen, konditionierten Reaktionen. Zum limbischen System unterhält er direkte reziproke Verbindungen. Er spielt eine evolutionär konservierte Schlüsselrolle bei der Inhibition des limbischen Systems und damit der Kontrolle der Furcht- und Angstentstehung. Eine ähnliche Verschaltung existiert auch zum Locus coeruleus. Der rechte PFC ist stärker an der Regulation emotionaler Reaktionen beteiligt als der linke PFC, der wiederum v. a. sprachliche Informationen sequenziell verarbeitet. Anteile des medialen PFC bewirken eine Down-Regulation der amygdalären Aktivität, hemmen konditionierte Angst und Furcht und steuern im Zusammenspiel mit dem Hippocampus einen Extinktionsprozess erlernter Angst- 
reaktionen (Koenigs \& Grafman 2009). Der dorsolaterale PFC ist in seinen Funktionen des Arbeitsgedächtnisses, der Reaktionsauswahl und der konkreten Reaktionsvorbereitung bedeutsam. Das anteriore Zingulum (ACC) hingegen reguliert den Grad und die Intensität des affektiven Disstresses, der durch eine emotionale Aktivierung ausgedrückt wird. Bei Angstreaktionen spielt weiterhin die anteriore Insel, die interozeptive Afferenzen aus dem Körperinneren versammelt, eine wesentliche Rolle (Craig 2009). Zusammen mit Informationen aus der Amygdala, dem Nucleus accumbens und dem orbitofrontalen Kortex generiert die anteriore Insel ein interozeptives Vorhersagesignal, das die Differenz zwischen dem aktuellen Körperzustand und dem erwarteten künftigen Zustand repräsentiert. Personen mit erhöhter Angstneigung zeichnen sich durch eine übertriebene aversive Erwartungshaltung aus, die wiederum leicht mit einem maladaptiven Vermeidungsverhalten einhergeht (Paulus \& Stein 2006). An der Generierung von länger anhaltender oder nicht prädizierbarer Angst scheinen insbesondere zusätzlich der Bettkern der Stria terminalis (BNST) und der Hippocampus involviert zu sein. Beider Funktionalität ist komplex. Innerhalb des dorsalen Anteils des BNST lösen Efferenzen aus dem ovalen BNST eher eine diffuse Angstspannung aus, während Efferenzen aus dem anterodorsalen BNST Angst reduzieren können. Der ventrale Anteil des BNST hingegen scheint in bedrohlichen Situationen über eine Aktivierung der Area tegmentalis ventralis (VTA) eine angstauslösende Wirkung zu haben, wobei auch die VTA hemmende und damit anxiolytische Efferenzen aufweist (Jennings et al. 2013, Kim et al. 2013).

Wichtige Erkenntnisse zu den differentiellen Einflüssen der am neuronalen Furcht-Angst-Regelkreis beteiligten Strukturen und Funktionssystemen stammen aus den diversen Neuroimaging-Verfahren (Fox \& Shackman 2017, Carlisi \& Robinson 2018).

\section{Neurotransmitter-/Rezeptormodelle der Angststörungen}

An der Vermittlung von Stress-, Angst- und Furchtreaktionen sind in einem ebenfalls höchst komplexen Zusammenspiel zahlreiche Neurotransmittersysteme beteiligt.

\section{GABA-System}

Bei den Angststörungen wird eine zentrale Dysregulation des Benzodiazepin-GABA-Rezeptorkomplexes diskutiert (Kalueff \& Nutt 2007). Die $\gamma$-Aminobuttersäure (GABA) ist der bedeutendste inhibitorische Neurotransmitter im ZNS mit einer intensiven Verteilung in der Großhirnrinde, in Teilen des limbischen Systems und im Kleinhirn. Zirka 1/3 aller Neuronen stehen unter GABAergen Einflüssen. Das Niveau der neuronalen Aktivität wird über die Balance zwischen der v. a. glutamaterg vermittelten Erregung und der GABAergen Hemmung reguliert. Eine zunehmende Verschiebung in Richtung GABA führt zu Sedierung, Amnesie und Ataxie, eine Verschiebung in Richtung Exzitation aber zu Angst, Unruhe, Schlaflosigkeit und erhöhter Anfallsneigung. Die meisten BenzodiazepinRezeptoren bzw. -bindungsstellen befinden sich am GABA-A-Rezeptorkomplex. Der experimentelle Einsatz von vollen und partiellen Benzodiazepin-Agonisten, von Antagonisten sowie von vollen und partiellen inversen Benzodiazepin-Agonisten hat zu einer sehr differenzierten Einsicht in die komplexe Funktionsweise dieses primären Hemmsystems im zentralen Nervensystem in ihrer ätiopathogenetischen Bedeutung für Angststörungen geführt (Jembrek \& Vlainic 2015).

\section{Glutamatsystem}

Auch die exzitatorischen Aminosäuren tragen bedeutsam zur Pathophysiologie von Angststörungen bei (Amiel \& Mathew 2007). Glutamat ist der wichtigste exzitatorische Neurotransmitter. Glutamat entfaltet seine Effekte an mehreren ionotropen und metabotropen Rezeptoren. Insbesondere der NMDA(N-Methyl-D-Aspartat)-Rezeptor-Subtypus befindet sich in einer homöostatischen Balance mit dem GABA-System. Er weist auch enge Interaktionen mit dem noradrenergen System auf. Die Angstkonditionierung steht zwar primär unter einem noradrenergen Einfluss, die Konsolidierung der konditionierten Furchtreaktion selbst wird aber wesentlich glutamaterg, speziell über Mechanismen der LongTerm Potentiation (LTP) vermittelt (Cortese \& Phan 2005). Auch Extinktionsvorgänge werden bedeutsam von der glutamatergen Neurotransmission beeinflusst (Davis et al. 2006; Simon \& Gorman 2006). Im gesamten Angst-Furcht-Regelkreis wird die glutamaterge Erregungausbreitung über spannungsabhängige Kalziumkanäle mitreguliert. Als ein erfolgreiches Behandlungsprinzip vor allem bei der generalisierten Angststörung hat sich der Einsatz von sogenannten $\alpha 2-\delta$ Liganden wie insbesondere von Pregabalin erwiesen, die bei stark aktivierten Kalziumkanälen eine übermäßige präsynaptische Freisetzung von Glutamat verhindern (Generoso et al. 2017).

\section{Noradrenerges System}

Die zentrale Rolle des noradrenergen Systems wird durch die bekannten somatischen Symptome bei Angstzuständen veranschaulicht, die über die noradrenergen postsynaptischen $\beta 1$-Rezeptoren vermittelt werden. Erhöhte Angst wie bei Panikattacken scheint mit einer exzessiven Freisetzung von Noradrenalin einherzugehen, was möglicherweise eine Dysfunktion der hemmenden $\alpha 2$-adrenergen Rezeptoren widerspiegelt. Unter wiederholter Einwirkung von Stressoren scheint es zu einer Sensitivierung der noradrenergen Antwort durch den Locus coeruleus zu kommen (Neumeister et al. 2005). Das GABAerge System projiziert stark auf den 
Locus coeruleus mit der größten Dichte an noradrenergen Neuronen im ZNS. Eine verringerte GABAerge Funktion könnte über eine Reduktion der inhibitorischen Effekte sekundär zu einer noradrenergen Hyperaktivität des Locus coeruleus beitragen. Die empirisch erprobte Wirksamkeit von Antidepressiva wie die SNRI oder NaSSA mit Effekten auch auf das noradrenerge System unterstreichen dessen pathogenetischen Stellenwert bei Angststörungen (Bandelow et al. 2017).

\section{Serotonerges System}

Eine bedeutsame modulatorische Rolle des serotonergen Systems in der Pathophysiologie von Angstzuständen wird vielfach nahegelegt. Unter den zahlreichen Serotoninrezeptorsubtypen spielt v. a. der präsynaptisch gelegene 5-HT1A-Autorezeptor, der G-Protein-gekoppelt bei Stimulation die Synthese und die Freisetzung von Serotonin hemmt, eine wichtige Rolle. Postsynaptisch übt der 5-HT1A-Rezeptor einen modulierenden Effekt auf die HPA-Achse aus. Patienten mit Panikstörung und sozialer Phobie weisen eine niedrigere Dichte von 5-HT1A-Rezeptoren in der Amygdala, den Raphekernen und im anterioren Zingulum auf (Lanzenberger et al. 2007). Anxiolytisch wirksame Substanzen wie Buspiron zeigen eine hohe Affinität gegenüber dem 5-HT1A-Rezeptor (Christmas et al. 2008). Sie stimulieren präsynaptisch, blockieren postsynaptisch und verringern sekundär die Dichte der 5-HT2-Rezeptoren. Die Bedeutung dieser Phosphoinositol verknüpften 5-HT2-Rezeptoren wird auch dadurch unterstrichen, dass die tertiären trizyklischen Antidepressiva (z. B. Imipramin), MAO-Hemmer und Trazodon als potente 5HT2A-Rezeptorantagonisten eine anxiolytischen Wirkung entfalten. Ferner scheinen auch 5-HT2C-Rezeptoren durch die SSRI und MAO-Hemmer bei Langzeitapplikation desensibilisiert $\mathrm{zu}$ werden. Auch der Natrium-Kanal-assoziierte 5-HT3-Rezeptor ist an der Angstregulation beteiligt (Olivier et al. 2000). Eine grundlegende Beteiligung des Serotoninsystems an der Neurobiologie der Angststörungen ist unbestreitbar. Dies bekräftigen nicht zuletzt auch die überzeugenden molekulargenetischen Befunde zur Assoziation mit dem funktionellen SERT-Polymorphismus (Houwing et al. 2017).

\section{Andere Neurotransmitter und Neuropeptide}

Neben diesen hervorgehobenen Neurotransmittersystemen spielen aber auch zahlreiche weitere Neurotransmitter, wie etwa Dopamin, Adenosin, Histamin, Opioide, Endocannabinoide eine differenzielle Rolle in der Vermittlung von Stressoren und Generierung von Angstaffekten. Zusammen mit Neuropeptiden (z.B. Neuropeptid Y und S), Stresshormonen, Neurotrophinen, Zytokinen und zellulären Mediatoren entfalten sie in Abhängigkeit von der Dauer, vom Kontext und der Art der Stressoren, dem Alter, dem Geschlecht und der genetischen Prädisposition hoch komplex orchestrierte Reaktionen (Joëls \& Baram 2009).

\section{Neuroendokrines Modell der Angststörungen}

Der stressinduzierten Freisetzung von CRH im Hypothalamus kommt eine zentrale pathogenetische Rolle in Angstzuständen zu. CRH erhöht nicht nur ACTH und Kortisol in der Stressantwort, sondern aktiviert im Locus coeruleus auch Noradrenalin und in den Raphekernen Serotonin. Hierbei sind jeweils reziproke Interaktionen $\mathrm{zu}$ beachten. Es existieren zwei Hauptrezeptortypen mit unterschiedlichen Funktionen. CRH1Rezeptoren vermitteln anxiogene, CRH2-Rezeptoren eher Angst mitigierende Effekte. Stressoren in der frühen Individualentwicklung können $\mathrm{zu}$ einer langfristigen Erhöhung von CRH und zu einer persistierenden Sensitivierung von CRH-haltigen Neuronen gegenüber im späteren Leben erfahrenen Stressoren führen (Dirven et al. 2017).

\section{Neuroinflammatorisches Modell der Angststörungen}

Bei Angststörungen können auch Veränderungen im inflammatorischen System nachgewiesen werden, wobei die kausale Richtung dieses Zusammenhangs weiter diskutiert wird. Vor allem bei Anzeichen einer chronischen „low-grade inflammation" infolge bedeutsamer Stressexpositionen können Einflüsse auf Angstvulnerabilität und Angstniveau angenommen werden. Eine komplexe Interaktion mit den weiteren Stressreaktionssystemen des sympathischen Nervensystems und der HPA-Achse sind hierbei zu beachten. Verstärkte Reagibilität der neuronalen Regelkreise von Bedrohung und Angst einerseits, abgeschwächte Reagibilität der neuronalen Regelkreise von Belohnung und von exekutiver Funktionalität andererseits sind spezielle Auswirkungen von stressinduzierten proinflammatorischen Prozessen (Nusslock \& Miller 2016; Felger 2018), mit weitreichenden Konsequenzen für assoziierte somatische Erkrankungsrisiken im langfristigen Verlauf von Angsterkrankungen (Kapfhammer 2018).

\section{Genetische und epigenetische Modelle der Angststörungen}

Ergebnisse der genetischen und epigenetischen Forschung haben in den letzten Jahrzehnten entscheidend zu einem besseren Verständnis der Vulnerabilität und Disposition zu Angststörungen beigetragen. Differenzielle Methoden fokussieren hierbei auf unterschiedliche Fragestellungen:

- „Häuft sich die Erkrankung in Familien?“ („Familialität") - Familienstudien

- „Was ist der ätiologische Beitrag von genetischen Faktoren?" („Heritabilität“) - Zwillings-/Adoptionsstudien

- „, Welche chromosomalen Loci, welche Gene sind involviert? " - Kopplungs-/Assoziationsstudien

- „Welche Gen-Umwelt-Interaktionen bestehen?" komplexe psychologische und molekulargenetische/ epigenetische Untersuchungsdesigns. 
Das Risiko für eine definierte Angststörung ist für Verwandte 1. Grades eines Angstpatienten signifikant erhöht: Panikstörung: OR (Odds-Ratio) $=5,0$; spezifische Phobien: $\mathrm{OR}=4,1$, generalisierte Angststörung: $\mathrm{OR}=6,1$ (Hettema et al. 2001). Die „Heritabilität“”, d. h. der Anteil genetischer Faktoren bei der Entstehung der Panikstörung liegt bei bis zu $43 \%$, die Heritabilitätswerte für die generalisierte Angststörung und die spezifischen Phobien bei ca. $30 \%$, für die soziale Phobie bei $51 \%$ und für die Agoraphobie bei $67 \%$ (Hettema et al. 2001). Die verbleibende Varianz jeweils wird durch individuelle Umweltfaktoren wie z. B. Lebensereignisse erklärt. Ein großer Varianzanteil bei Angststörungen betrifft folglich spezifische Umweltfaktoren bei einer Person (Hettema et al. 2005).

Analysiert man die zugrunde liegende Struktur von genetischen Einflüssen und Umweltrisikofaktoren bei Angststörungen, scheinen zunächst zwei dichotome genetische Faktoren die Panikstörung, die Agoraphobie und die generalisierte Angststörung einerseits, die spezifischen Phobien andererseits am besten zu erklären, wobei die soziale Phobie eine Zwischenstellung einnimmt (Kendler et al. 2001). Ein gemeinsamer Faktor einer geteilten Umwelt erklärt weniger als $12 \%$ der Varianz in den einzelnen Angststörungen. Er bedeutet aber für einige Angstaspekte, wie z. B. den Symptomen von Trennungsangst, dass dem Modelllernen an den Eltern in der Kernfamilie durchaus eine besondere Rolle zukommen kann (Eley 2009). Das genetische Risiko für Angststörungen folgt nicht einem Erbgang nach Mendelschen Regeln, sondern dem Muster einer Vererbung in einem polygenetischen Modell. Dementsprechend spricht man im Zusammenhang von komplex-genetischen Erkrankungen wie den Angststörungen - im Gegensatz zu „kausalen Genen“ bei monogenetisch bedingten Erkrankungen - von „Vulnerabilitätsgenen“ oder „Risikogenen“, die in individueller Kombination und/oder Wechselwirkung („Epistase“) miteinander oder mit Umweltfaktoren („Epigenese“) zur Pathogenese der Erkrankung beitragen. Geschlechtsspezifische genetische Effekte werden für die Agoraphobie, soziale Phobie, Situationsphobien und Blut-Verletzungs-Phobie angenommen, nicht aber für Tierphobien (Kendler et al. 2002).

Entsprechend einem polygenen Erbgang konzentrieren sich molekulargenetische Untersuchungen in den letzten Jahren darauf, Assoziationen einerseits für differenzielle klinische Unterformen von Angststörungen, andererseits für gemeinsam von allen Angststörungen geteilte Vulnerabilitäten („Endophänotypen“ oder „intermediäre Phänotypen“) aufzudecken (Canli et al. 2009). Lediglich letztere allgemeine Aspekte sollen hier kurz angeführt werden.

Eine Strategie, die genetische Basis einer allgemeinen Ängstlichkeit näher zu charakterisieren, ist angesichts der hohen Koexistenz (sequentiell in der Entwicklung und parallel als Komorbidität) einzelner Angststörungen in individuellen Krankheitsverläufen von hoher Bedeutung (Hettema et al. 2006a). Ein solcher allgemeinerer Suszeptibilitätslocus wurde etwa auf dem Chromosom 4q21 beschrieben, in dessen Nähe auch das Neuropeptid Y kodiert wird (Kaabi et al. 2006). Das Glutaminsäuredekarboxylase-Gen1 könnte ebenfalls eine solche Rolle spielen (Hettema et al. 2006b). Der Temperamentsphänotyp „Verhaltenshemmung“, der etwa $10-15 \%$ der US-Kinder betrifft, sowohl behavioral als auch biologisch hoch reliabel zu charakterisieren ist und eine ca. 70\%-ige Heritabilität aufweist, war in einer familiengestützten Assoziationsanalyse mit Polymorphismen im CRH-Gen assoziiert (Smoller et al. 2005). Lesch et al. (1996) gelang erstmals der Nachweis einer signifikanten Assoziation der in der Persönlichkeitsdimension „Neurotizismus“ mitenthaltenen „Trait-Ängstlichkeit“" mit dem kurzen Allel eines funktionellen Polymorphismus im Serotonin-Transporter-Gen (SERT).

Zunehmend werden in sog. Imaging-Genetics-Studien neurale Aktivierungsmuster als intermediäre Phänotypen von psychischen Erkrankungen hinsichtlich ihrer genetischen Grundlage untersucht (Domschke \& Dannlowski 2010). So wurde eine allgemeine Bedeutung des SERT-Polymorphismus für die emotionale Regulation und soziale Kognition vorgeschlagen (Canli \& Lesch 2007). Hariri et al. (2002, 2005) fanden, dass SERT-,,short“-Allel-Träger auf angsterfüllte bzw. furchterregende Gesichter verstärkte Amygdala-vermittelte Furchtreaktionen im fMRT zeigen. Pezewas et al. (2005) konnten nachweisen, dass das SERT-,,short"Allel für die Entkoppelung des amygdalären Regelkreises von Steuereinflüssen des präfrontalen Kortex bzw. des anterioren Zingulums mit verantwortlich ist. Künftige Forschungsansätze werden weiter das $\mathrm{Zu}$ sammenspiel von genetischen Dispositionen und Umweltstressoren in kritischen Entwicklungsabschnitten analysieren und so zu einem bedeutsamen Erkenntniszugewinn für die Entstehung von Angststörungen kommen. Studien zeigten z. B. einen Einfluss des SERT-,,short"-Allels in Interaktion mit belastenden oder traumatischen Kindheitserlebnissen auf das Risiko für eine erhöhte Angstsensitivität bei gesunden Probanden (Klauke et al. 2011).

Epigenetischen Prozessen wie z. B. der DNA-Methylierung/Demethylierung als flexiblen, zeitlich sehr dynamischen Mechanismen in der Steuerung der Genexpression kommt in künftigen Forschungen eine wachsende Bedeutung zu. Epigenetische Ergebnisse besitzen eine Relevanz für das Verständnis von Verläufen und die Kontrolle der Wirksamkeit von Therapien (Schübel et al. 2016; Schiele \& Domschke 2018, Ziegler \& Domschke 2018).

Neben diesen geschilderten neurobiologischen und genetischen/epigenetischen Befunden, die sich auf allgemeine Aspekte der Neurobiologie der Angststörungen beziehen, sind weiter auch zahlreiche differenzierende Ergebnisse zu speziellen einzelnen Angsterkrankungen wie z.B. zur Panikstörung, Generalisierten Angststörung oder sozialen Phobie festzuhalten (Domschke et al. 2017). 


\section{GRUNDZÜGE DER BEHANDLUNG VON ANGSTSTÖRUNGEN}

Die Therapie von Angsterkrankungen umfasst psychopharmakologische und psychotherapeutische Ansätze. Ein Evidenz-basiertes Vorgehen kann beispielsweise der deutschen S3-Leitlinie der DGPPN entnommen werden (Bandelow et al. 2015a; Ströhle et al. 2018). Grundsätzlich sollen Patienten - mit Ausnahme der Patienten mit spezifischen Phobien - sowohl psychotherapeutische als auch medikamentöse Ansätze im Rahmen einer grundlegenden Psychoedukation als Therapieoptionen vorgestellt werden. Patientenpräferenzen für eine dieser Hauptbehandlungsformen sind zu berücksichtigen. Eine fachlich fundierte Entscheidung sollte stets konsensuell erfolgen, sich bei einer vorliegenden Angststörung nach Schwere der Symptomatik, Verlaufsdauer, bestehender psychischer und somatischer Komorbidität, hiermit verbundener subjektiver Belastung und psychosozialer Beeinträchtigung sowie nach der Verfügbarkeit einer favorisierten Therapieform richten. Eine Aufklärung über die wahrscheinliche Dauer bis zu einem spürbaren Wirkeintritt und die Nachhaltigkeit der erzielten Effekte, über grundlegende Nebenwirkungen und die empfohlene Zeitdauer der gewählten Therapieform muss erfolgen. Es ist über einen eventuellen Wechsel der aufgenommenen Therapiemodalität bei Nichtwirksamkeit, über das Procedere in Notfällen, den Sinn einer Kombination von medikamentösen und psychotherapeutischen Ansätzen zu sprechen.

Für psychopharmakologische Ansätze bei Angststörungen existieren zahlreiche Präparate aus einer Reihe unterschiedlicher Substanzklassen, denen eine gute empirische Wirksamkeit bescheinigt werden kann. Den SSRI und den SNRI kommt der Status von Medikamenten der 1. Wahl sowohl bei der Panikstörung, der sozialen Phobie und der generalisierten Angststörung zu. Bei der GAD stellt das Pregabalin eine gleichwertige Therapieoption dar. Alle anderen in der Behandlung der einzelnen Angststörungen untersuchten Medikamente sind in der Evidenz- bzw., bei Berücksichtigung des vorliegenden Nebenwirkungsspektrums, der Empfehlungsgraduierung nachgeordnet. Der Einsatz von Benzodiazepinen wird trotz guter Wirkbelege auf Grund der Nebenwirkungen, insbesondere des Gewöhnungs- und möglichen Abhängigkeitsrisikos kritisch gesehen, sinnvoll auf die definierte Zeitspanne der Einstellung auf serotonerge Antidepressiva beschränkt, nur in wohl reflektierten und dokumentierten Einzelfällen aber auch als für eine Langzeitapplikation vertretbar angesehen. Selbst bei einer Entscheidung für eine bevorzugte Psychopharmakotherapie müssen stets wichtige psychoedukaktive und supportiv-psychotherapeutische Elemente in die individuelle Behandlung eines Angstpatienten integriert werden. Eine Behandlung von Angststörungen mit Medikamenten ist wegen der häufig chronischen Verläufe sehr oft auf eine Langzeitperspektive hin anzulegen. $\mathrm{Zu}$ schnelle Reduktionsschritte oder das Absetzen einer wirksam etablierten
Medikation sind mit einem bedeutsamen Risiko von Verschlechterungen in der Angstkontrolle oder von Rezidiven der Angststörung verbunden (Batelaan et al. 2017). Dieser empirische Sachverhalt einer oft notwendigen Langzeitmedikation stellt große Herausforderungen sowohl an die Therapieadhärenz des Patienten als auch an die Bemühungen des Arztes um die Aufrechterhaltung einer verlässlichen therapeutischen Beziehung.

Im Vergleich von Psychotherapie und Pharmakotherapie werden für die psychotherapeutischen Ansätze bei den Angststörungen häufig höhere Effektstärken gefunden (Roth \& Fonagy 2005). Nicht selten ist hierbei aber die Qualität des medikamentösen Therapiearms in den Untersuchungen zu kritisieren. Ferner wurden in den Studiensamples mehrheitlich Patienten eingeschlossen, die ohne psychische Komorbidität waren und ein nur geringgradiges agoraphobisches Verhalten zeigten. Gegenüber früheren Einschätzungen relativieren komplexere metaanalytische Untersuchungen diese Aussagen bedeutsam (Pompoli et al. 2016, Carpenter et al. 2018). Vor allem im Hinblick auf eine in den Psychotherapiestudien übliche Auswahl von Patienten ohne begleitende psychische Komorbiditäten sollten die berichteten Effizienzgrade nicht überschätzt und auch nicht so ohne Weiteres auf durchschnittliche Versorgungsbedingungen extrapoliert werden (Westen \& Morrison 2001, Morrison et al. 2003, Bandelow et al. 2015a). Kognitiv-verhaltenstherapeutische Ansätze können sich auf die umfangsreichste empirische Datenbasis mit überzeugenden Belegen für eine gute Wirksamkeit stützen. Sie werden in den unterschiedlichen Guidelines meist als Psychotherapieansatz der 1. Wahl eingestuft (Bandelow et al. 2015a; Ströhle et al. 2018). Von anderen Arbeitsgruppen wird in differenzierten metaanalytischen Studien eine Gleichwertigkeit der Wirksamkeit von störungsorientierten psychodynamischen Verfahren diskutiert (Steinert et al. 2017).

Nicht selten legen Komplexität und Chronizität entweder simultan-überlappend oder sequentiell eine Kombination von medikamentösen und psychologischpsychotherapeutischen Verfahren als sinnvoll und vorteilhaft nahe (Craske \& Stein 2016). Es lassen sich mittlerweile empirische Belege für eine solche Kombinationsstrategie vorlegen, wie eine Metaanalyse zeigte (Bandelow et al. 2015b).

\section{SCHLUSSBEMERKUNG}

Angststörungen zählen zu den häufigsten psychischen Störungen in der Allgemeinbevölkerung. Sie sind mit einem hohen subjektiven Leiden einer oft chronischen Persistenz, mit zusätzlichen komorbiden anderen psychischen und auch somatischen Krankheitsrisiken und hieraus resultierend mit zahlreichen psychosozialen Behinderungsgraden und großer sozioökonomischer Belastung verbunden. Angststörungen werden heute prinzipiell innerhalb eines multifaktoriellen, biopsychosozialen Krankheitsmodells konzipiert. Psychologische, psychosoziale, aber vor allem auch neurobiologische 
und genetische Forschungsansätze haben zu einem vertieften und komplexeren Verständnis der mannigfaltigen Einflüsse auf ihre Entstehung, Auslösung und Aufrechterhaltung geführt. Angststörungen können sowohl psychopharmakologisch als auch psychotherapeutisch wirksam behandelt werden. In einer Gesamtbeurteilung kann festgehalten werden, dass die große Mehrheit der unkomplizierten spezifischen Phobien und Panikstörungen gut und sehr wahrscheinlich auch anhaltend mit Psychotherapie gebessert werden können. Generalisierte Angststörungen und soziale Phobien benötigen hingegen häufig längerfristige störungsorientierte Psychotherapien. Schwere Angststörungen aber, vor allem bei chronischen Verläufen, mit zusätzlichen psychischen und somatischen Komplikationen und ausgeprägter psychosozialer Beeinträchtigung / Behinderung sollten zusätzlich medikamentös behandelt werden.

\section{Acknowledgements: None.}

Conflict of interest: None to declare.

\section{Contribution of individual authors:}

Equivalent contributions of both authors.

\section{Literatur}

1. Amiel JM, Mathew SJ: Glutamate and anxiety disorders. Curr Psychiatry Rep 2007; 9:278-283

2. Arnsten AFT: Stress signalling pathways that impair prefrontal cortex structure and function. Nat Rev Neurosci 2009; 10:410-422

3. Asmundson GJ, Taylor S, Smits JA: Panic disorder and agoraphobia: an overview and commentary on DSM-5 changes. Depress Anxiety 2014; 31:480-486

4. Bandelow B, Lichte T, Rudolf $S$, Wiltink J, Beutel ME: The German guidelines for the treatment of anxiety disorders. Eur Arch Psychiatry Clin Neurosci 2015a; 265:363-73

5. Bandelow B, Michaelis $S$, Wedekind D: Treatment of anxiety disorders. Dialogues Clin Neurosci 2017; 19:93-107

6. Bandelow B, Reitt M, Röver C, Michaelis S, Görlich Y, Wedekind D: Efficacy of treatments for anxiety disorders: a meta-analysis. Int Clin Psychopharmacol 2015b; 30:183-192

7. Batelaan NM, Bosman RC, Muntingh A, Scholten WD, Huijbregts KM, van Balkom AJLM: Risk of relapse after antidepressant discontinuation in anxiety disorders, obsessive-compulsive disorder, and post-traumatic stress disorder: systematic review and meta-analysis of relapse prevention trials. BMJ 2017; 358:j3927

8. Canli T, Ferri J, Duman EA: Genetics of emotion regulation. Neuroscience 2009; 164:43-54

9. Canli T, Lesch KP: Long story short: the serotonin transporter in emotion regulation and social cognition. Nat Neurosci 2007; 10:1103-1109

10. Canuto A, Weber K, Baertschi M, et al.: Anxiety disorders in old age: psychiatric comorbidities, quality of life, and prevalence according to age, gender, and country. Am J Geriatr Psychiatry 2018; 26:174-185
11. Carlisi CO, Robinson OJ: The role of prefrontal-subcortical circuitry in negative bias in anxiety: Translational, developmental and treatment perspectives. Brain Neurosci Adv 2018; 2:2398212818774223

12. Carpenter JK, Andrews LA, Witcraft SM, Powers MB, Smits JAJ, Hofmann SG: Cognitive behavioral therapy for anxiety and related disorders: A meta-analysis of randomized placebo-controlled trials. Depress Anxiety 2018; 35:502-514

13. Christmas D, Hood S, Nutt D: Potential novel anxiolytic drugs. Curr Pharm Des 2008; 14:3534-3546

14. Cisler JM, Olatunji BO, Lohr JM: Disgust, fear, and the anxiety disorders: a critical review. Clin Psychol Rev 2009; 29:34-46

15. Cortese BM, Phan KL: The role of glutamate in anxiety and related disorders. CNS Spectr 2005; 10:820-830

16. Craig BAD: How do you feel - now? The anterior insula and human awareness. Nat Rev Neurosci 2009; 10:5970

17. Craske MG, Stein MB, Eley TC, Milad MR, Holmes A, Rapee RM, Wittchen HU: Anxiety disorders. Nat Rev Dis Primers 2017; 3:17024

18. Davis M, Myers KM, Chhatwal J, Ressler KJ: Pharmacological treatments that facilitate extinction of fear: relevance to psychotherapy. NeuroRx 2006; 3:82-96

19. Davis M, Walker DL, Miles L, Grillon C: Phasic vs sustained fear in rats and humans: role of the extended amygdala in fear vs anxiety. Neuropsychopharmacology 2010; 35:105-135

20. Dirven BCJ, Homberg JR, Kozicz T, Henckens MJAG: Epigenetic programming of the neuroendocrine stress response by adult life stress. J Mol Endocrinol 2017; 59:R11-R31

21. Domschke K, Dannlowski U: Imaging genetics of anxiety disorders. Neuroimage 2010; 53:822-831

22. Domschke K, Kapfhammer HP, Deckert J: Angststörungen. In: Möller HJ, Laux G, Kapfhammer HP (Hrsg) Psychiatrie, Psychosomatik und Psychotherapie. 5. Aufl. Springer Verlag GmbH Deutschland 2017; Bd. 3, 1857-1932

23. Eaton WW, Bienvenu OJ, Miloyan B: Specific phobias. Lancet Psychiatry 2018; 5:678-686

24. Eley TC: The genetic basis of anxiety disorders. In: Andrews G, Charney DS, Sirovatka PJ, Regier DA (Hrsg) Stress-induced and fear circuitry disorders. Advancing the research agenda for DSM-V. Amer Psychiatric Press, Inc, Washington, DC, 2009; 145-158

25. ESEMeD/MHEDEA 2000 Investigators: Prevalence of mental disorders in Europe: results from the European Study of the Epidemiology of Mental Disorders (ESEMeD) project. Acta Psychiatr Scand 2004; 109(Suppl 420):21-27

26. Felger JC: Imaging the role of inflammation in mood and anxiety-related disorders. Curr Neuropharmacol 2018; 16:533-558

27. Fox AS, Shackman AJ: The central extended amygdala in fear and anxiety: Closing the gap between mechanistic and neuroimaging research. Neurosci Lett 2017; pii: S0304-3940(17)30965-5

28. Geiger M, Neufang S, Stein DJ, Domschke K: Arousal and the attentional network in panic disorder. Hum Psychopharmacol 2014; 29:599-603

29. Generoso MB, Trevizol AP, Kasper S, Cho HJ, Cordeiro Q, Shiozawa P: Pregabalin for generalized anxiety disorder: an updated systematic review and meta-analysis. Int Clin Psychopharmacol 2017; 32:49-55 
30. Goodwin H, Yiend J, Hirsch CR: Generalized Anxiety Disorder, worry and attention to threat: A systematic review. Clin Psychol Rev 2017; 54:107-122

31. Grant BF, Hasin D, Blanco C et al.: The epidemiology of social phobia in the United States: results from the National Epidemiologic Survey on Alcohol and Related Conditions. J Clin Psychiatry 2005; 66:1351-1361

32. Grant BF, Hasin DS, Stinson FS et al.: The epidemiology of DSM-IV panic disorder and agoraphobia in the United States: results from the National Epidemiologic Survey on Alcohol and Related Conditions. J Clin Psychiatry 2006; 67:363-374

33. Hariri AR, Drabant EM, Munoz KE et al.: A susceptibility gene for affective disorders and the response of the human amygdala. Arch Gen Psychiatry 2005; 62:146-152

34. Hariri AR, Mattay VS, Tessitore A et al.: Serotonin transporter genetic variation and the response of the human amygdala. Science 2002; 297:400-403

35. Hettema JM, An SS, Neale MC et al.: Association between glutamic acid decarboxylase genes and anxiety disorders, major depression, and neuroticism. Mol Psychiatry 2006a; 11:752-762

36. Hettema JM, Neale MC, Kendler KS: A review and metaanalysis of the genetic epidemiology of anxiety disorders. Am J Psychiatry 2001; 158:1568-1578

37. Hettema JM, Neale MC, Myers JM et al.: A population-based twin study of the relationship between neuroticism and internalizing disorders. Am J Psychiatry 2006b; 163:857-864

38. Hettema JM, Prescott CA, Myers JM et al.: The structure of genetic and environmental risk factors for anxiety disorders in men and women. Arch Gen Psychiatry 2005; 62:182-189

39. Houwing DJ, Buwalda B, van der Zee EA, de Boer SF, Olivier JDA: The serotonin transporter and early life stress: Translational perspectives. Front Cell Neurosci 2017:11:117

40. Jembrek MJ, Vlainic J: GABA Receptors: pharmacological potential and pitfalls. Curr Pharm Des 2015, 21:4943-4959

41. Jennings JH, Sparta DR, Stamatakis AM, Ung RL, Pleil KE, Kash TL, Stuber GD: Distinct extended amygdala circuits for divergent motivational states. Nature 2013; 496:224-228

42. Kaabi B, Gelernter J, Woods SW et al.: Genome scan for loci predisposing to anxiety disorders using a novel multivariate approach: strong evidence for a chromosome 4 risk locus. Am J Hum Genet 2006; 78:543-553

43. Kalueff $A V$, Nutt DJ: Role of GABA in anxiety and depression. Depress Anxiety 2007; 24:495-517

44. Kapfhammer HP: Acute and long-term mental and physical sequelae in the aftermath of traumatic exposuresome remarks on "the body keeps the score". Psychiatr Danub 2018; 30:252-270

45. Kapfhammer HP: Psychische Störungen bei somatischen Krankheiten. In: Möller HJ, Laux G, Kapfhammer HP (Hrsg) Psychiatrie, Psychosomatik und Psychotherapie. 5. Aufl. Springer Verlag GmbH Deutschland 2017; Bd. 4, 2693-2805

46. Kendler KS, Jacobson KC, Myers J, Prescott CA: Sex differences in genetic and environmental risk factors for irrational fears and phobias. Psychol Med 2002; 32:209-217

47. Kendler KS, Myers J, Prescott CA, Neale MC: The genetic epidemiology of irrational fears and phobias in men. Arch Gen Psychiatry 2001; 58:257-265
48. Kessler RC, Berglund P, Demler $O$ et al.: Lifetime prevalence and age-of-onset distributions of DSM-IV disorders in the National Comorbidity Survey Replication. Arch Gen Psychiatry 2005; 62:593-602

49. Kessler RC, Ruscio AM, Shear K, Wittchen HU: Epidemiology of anxiety disorders. In: Anthony MM, Stein MB (eds) Oxford handbook of anxiety and related disorders. Oxford library of psychology. Oxford University Press, Oxford 2009; 19-33

50. Kim SY, Adhikari A, Lee SY, Marshel JH, Kim CK, Mallory CS, Lo M, Pak S, Mattis J, Lim BK, Malenka RC, Warden MR, Neve R, Tye KM, Deisseroth K: Diverging neural pathways assemble a behavioural state from separable features in anxiety. Nature 2013; 496:219-223

51. Klauke B, Deckert J, Reif A, Pauli P, Zwanzger P, Baumann C, Arolt V, Glöckner-Rist A, Domschke K: Serotonin transporter gene and childhood trauma - a GxE effect on anxiety sensitivity. Depress Anxiety 2011; 28:1048-1057

52. Koenigs M, Grafman J: Posttraumatic stress disorder: the role of medial prefrontal cortex and amygdale. Neuroscientist 2009; 15:540-548

53. Kogan CS, Stein DJ, Maj M, First MB, Emmelkamp PM, Reed GM: The classification of anxiety and fear-related disorders in the ICD-11. Depress Anxiety 2016; 33:1141-1154

54. Kossowsky J, Pfaltz MC, Schneider S, Taeymans J, Locher $C$, Gaab J: The separation anxiety hypothesis of panic disorder revisited: a meta-analysis. Am J Psychiatry 2013; 170:768-781

55. Lanzenberger RR, Mitterhauser $M$, Spindelegger $C$ et al.: Reduced serotonin-1A receptor binding in social anxiety disorder. Biol Psychiatry 2007; 61:1081-1089

56. LeDoux JE, Pine DS: Using neuroscience to help understand fear and anxiety: a two-system framework. Am J Psychiatry 2016; 173:1083-1093

57. Leichsenring F, Leweke F: Social anxiety disorder. N Engl $J$ Med 2017; 376:2255-2264

58. Lesch KP, Bengel D, Heils A et al.: Association of anxietyrelated traits with a polymorphism in the serotonin transporter gene regulatory region. Science 1996; 274:1527-1531

59. Lieb R, Becker E, Altamura C: The epidemiology of generalized anxiety disorder in Europe. Eur Neuropsychopharmacol 2005; 15:445-452

60. Lijster JM, Dierckx B, Utens EM, Verhulst FC, Zieldorff $C$, Dieleman GC, Legerstee JS: The age of onset of anxiety disorders. Can J Psychiatry 2017; 62:237-246

61. Locke AB, Kirst N, Shultz CG: Diagnosis and management of generalized anxiety disorder and panic disorder in adults. Am Fam Physician 2015; 91:617-24

62. McNally RJ: Choking phobia: a review of the literature. Compr Psychiatry 1994; 35:83-89

63. Montag C, Panksepp J: Primary emotional systems and personality: an evolutionary perspective. Front Psychol 2017; 8:464

64. Morrison KH, Bradley R, Westen D: The external validity of controlled clinical trials of psychotherapy for depression and anxiety: a naturalistic study. Psychol Psychother 2003; 76:109-132

65. Murray CJ, Abraham J, Ali MK, Alvarado M, Atkinson C, Baddour LM: US Burden of Disease Collaborators et al. The state of US health, 1990-2010: burden of diseases, injuries, and risk factors. JAMA 2013; 310:591-608

66. Neumeister A, Daher RJ, Charney DS: Anxiety disorders. noradrenergic neurotransmission. Handb Exp Pharmacol $2005 ; 169: 205-223$ 
67. Nusslock R, Miller GE: Early-life adversity and physical and emotional health across the lifespan: a neuro-immune network hypothesis. Biol Psychiatry 2016; 80:23-32

68. Olivier B, van Wijngaarden I, Soudijn W: 5-HT3 receptor antagonists and anxiety: a preclinical and clinical view. Eur Neuropsychopharmacol 2000; 10:77-95

69. Oyebode F: Sim's symptoms in the mind. An introduction to descriptive psychopathology, 5th ed. Saunders, Elsevier, Edinburgh/London/New York, 2014

70. Patel V, Chisholm D, Parikh R, et al.: Addressing the burden of mental, neurological, and substance use disorders: key messages from Disease Control Priorities, 3rd edition. Lancet 2016; 387:1672-85

71. Paulus MP, Stein MB: An insular view of anxiety. Biol Psychiatry 2006; 60:383-387

72. Pezewas L, Meyer-Lindenberg A, Drabant EM et al.: 5-HTTLPR polymorphism impacts human cingulateamygdala interactions: a genetic susceptibility mechanism for depression. Nat Neurosci 2005; 8:828-834

73. Pompoli A, Furukawa TA, Imai H, Tajika A, Efthimiou $O$, Salanti G: Psychological therapies for panic disorder with or without agoraphobia in adults: a network metaanalysis. Cochrane Database Syst Rev 2016; 4: $C D 011004$

74. Rees CS, Richards JC, Smith LM: Medical utilisation and costs in panic disorder: a comparison with social phobia. J Anxiety Disord 1998; 12:421-35

75. Roth A, Fonagy P: What works for whom? A critical review of psychotherapy research, 2nd ed. Guilford, New York/London, 2005

76. Sartorius N, Ustun TB, Lecrubier $U$ et al.: Depression comorbid with anxiety: results from the WHO study on psychological disorders in primary health care. $\mathrm{Br} J$ Psychiatry 1996; 168:38-43

77. Schiele MA, Domschke K: Epigenetics at the crossroads between genes, environment and resilience in anxiety disorders. Genes Brain Behav 2018; 17:e12423

78. Schübel K, Gitik M, Domschke K, Goldman D: Making sense of epigenetics. Int J Neuropsychopharmacol 2016; 19:1-10

79. Shear K, Jin R, Ruscio AM, Walters EE, Kessler RC: Prevalence and correlates of estimated DSM-IV child and adult separation anxiety disorder in the National Comorbidity Survey Replication. Am J Psychiatry 2006; 163:1074-1083

80. Simon AB, Gorman JM: Advances in the treatment of anxiety: targeting glutamate. NeuroRx 2006; 3:57-68
81. Smoller JW, Yamaki LH, Fagerness JA et al.: The corticotropin releasing hormone gene and behavioral inhibition in children at risk for panic disorder. Biol Psychiatry 2005; 57:1485-1492

82. Steel $Z$ et al.: The global prevalence of common mental disorders: a systematic review and meta-analysis 1980 2013. Int J Epidemiol 2014; 43:476-493

83. Stein DJ, Scott KM, de Jonge P, Kessler RC: Epidemiology of anxiety disorders: from surveys to nosology and back. Dialogues Clin Neurosci 2017; 19:127-136

84. Steinert C, Munder T, Rabung S, Hoyer J, Leichsenring F: Different standards when assessing the evidence for psychodynamic therapy? Response to Cristea et al. Am J Psychiatry 2017; 174:1123-1124

85. Stinson FS, Dawson DA, Chou PS et al.: The epidemiology of DSM-IV specific phobia in the USA: results from the National Epidemiologic Survey on Alcohol and Related Conditions. Psychol Med 2007; 37:1047-1059

86. Ströhle A, Gensichen J, Domschke K: The diagnosis and treatment of anxiety disorders. Dtsch Arztebl Int 2018; 155:611-620

87. Tolin DF, Gilliam CM, Dufresne D: The economic and social burden of anxiety disorders. In: Stein DJ, Hollander, Rothbaum BO (eds) Textbook of anxiety disorders. American Psychiatric Publishing, Washington, DC/London 2009; 731-746

88. Ulrich-Lai YM, Herman JP: Neural regulation of endocrine and autonomic stress responses. Nat Rev Neurosci 2009; 10:397-409

89. Westen D, Morrison K: A multidimensional meta-analysis of treatments for depression, panic, and generalized anxiety disorder: an empirical examination of the status of empirically supported therapies. J Consult Clin Psychol 2001: 69:875-899

90. Wittchen HU, Jacobi F, Rehm J, Gustavsson A, Svensson M, Jonsson B, Olesen J, Allgulander C, Alonso J, Faravelli C, Fratiglioni L, Jennum $P$, Lieb $R$, Maercker A, van $O s J$, Preisig M, Salvador-Carulla L, Simon R, Steinhausen HC: The size and burden of mental disorders and other disorders of the brain in Europe 2010. Eur Neuropsychopharmacol 2011; 21:655-79

91. Wittchen $H U$, Reed $V$, Kessler RC: The relationship of agoraphobia and panic in a community sample of adolescents and young adults. Arch Gen Psychiatry 1998; 55:1017-1024

92. Ziegler $C$, Domschke K: Epigenetic signature of MAOA and $M A O B$ genes in mental disorders. $J$ Neural Transm (Vienna) 2018 Sep 21; [Epub ahead of print]

Correspondence:

Univ. Prof. Hans-Peter Kapfhammer, MD, PhD

Klinik für Psychiatrie und Psychotherapeutische Medizin,

Medizinische Universität Graz

Auenbruggerplatz 31, A-8036 Graz, Austria

E-mail: Hans-peter.kapfhammer@klinikum-graz.at 